\title{
STRATEGIC INTERACTION AND CATCHING UP
}

\author{
Mehmet Özer and Çağrı Sağlam \\ Department of Economics, Bilkent University, Turkey
}

\begin{abstract}
In this study, we prove that the strategic interaction among agents differing in initial wealth levels leads the poor to be able to catch up with the rich, which is not the case for the standard Ramsey model where the initial wealth differences perpetuate. Extending the analysis to account for relative wealth concern and the adjustment cost of consumption, the strategic interaction among agents is shown to affect not only the distribution of wealth in the long run but also the transitional dynamics substantially. In particular, we show that structurally very simple frameworks may lead to limit cycles thanks to the strategic interaction among agents in the economy.
\end{abstract}

Keywords: catching up, Hopf bifurcation, open-loop Nash equilibrium, Ramsey model

JEL classification numbers: C73, D31, O41

\section{INTRODUCTION}

The question of catching-up has always been one of the main concerns of macroeconomics. Stiglitz (1969) has shown that the poor will be able to catch up with the rich in a Solow economy. This analysis rests on the assumption that agents do not save optimally. In contrast with this, in a dynamic general equilibrium model $\grave{a}$ la Ramsey-Cass-Koopmans, Kemp and Shimomura (1992) has shown that if all households have the same time-preference rate, then the distribution of wealth will be history dependent so that the initial wealth inequality will persist even in the long run. However, in all of these studies, agents are thought to have no power in influencing the performance of aggregate economy and act as a price taker on all markets in a competitive equilibrium. Knowing that the number of households is finite, this contradicts with the rationality of the agents in the economy (see Pichler and Sorger, 2009). Moreover, the fact that social or economic similarities enforce individuals to constitute a small number of powerful groups and agents belonging to the same economic classes show similar tendencies in choosing their decision variables, makes the consideration of the strategic interaction among agents inevitable. ${ }^{1}$

Correspondence: Mehmet Özer, Department of Economics, Bilkent University, 06800 Ankara, Turkey. E-mail: ozerm@bilkent.edu.tr.

${ }^{1}$ Thanks to the comment of an anonymous referee, consider as an example the labor owned enterprises in accordance with the Action Programme (1989) of the European Commission (see Guadona, 2008). Given their limited number and heterogeneity in terms of initial asset and share holdings, workers that own a share of the firm may realize their market power and act strategically in choosing their capital paths. Also for the emerging recognition of strategic interaction in growth theory, see among others, Fershtman and Muller 
The objective of this paper is to analyze how the dynamic strategic interactions among agents affect the long-run distribution of wealth in terms of catching up and the transitional dynamics in an economy. In particular, we analyze to what extent the strategic interaction inherent in agents' strategies can alter the results on catching up that could have emerged under a competitive equilibrium framework and try to answer the following questions: Can a rich household with a larger initial stock credibly maintain this advantage to preempt the rival's accumulation and reach a better long-run stock of capital? Put differently, can this kind of initial advantage vanish in the non-cooperative equilibrium of this class of games even with open-loop strategies?

To do so, we consider a strategic Ramsey model in which finitely many households differ only in terms of their initial wealth. The households no longer act as price-takers but they take into account the effects of their accumulation decisions on market prices. Taking into account the inverse factor demand functions, the households play a Nash equilibrium by choosing their capital paths. We assume that the households employ open-loop strategies so that they give their accumulation decisions as simple time paths and commit themselves to stick to these preannounced paths as equilibrium strategies (see e.g. Sorger, 2002, 2008; Bethmann, 2008).

In this setup, households choose their strategies simultaneously and each household is faced with a single criterion optimization problem constrained by the strategies of the rivals taken as given. In this respect, adopting open-loop strategies reflect the slightest departure from the competitive equilibrium framework as it does not allow for genuine interaction between players during the game (see Sorger, 2008; Camacho et al., 2013). However, even under this small departure from the competitive equilibrium framework, we show that considering the strategic interaction among agents in the economy changes the qualitative properties of the standard Ramsey model drastically. ${ }^{2}$

In the absence of strategic interaction, the poor will never be able to catch up with the rich as pointed out in Van Long and Shimomura (2004). However, incorporating the strategic behavior among agents leads to the wealth level of the initially poor and the rich households to be the same at the stationary state. We extend our analysis on the dynamic implications of strategic interaction, to account for relative wealth concern (capitalist spirit ${ }^{3}$ ) and adjustment cost of consumption on utility. Deviating from the competitive equilibrium framework, we show that the strategic interaction among agents in the economy leads to a change not only in the distribution of wealth in the long run but also in the transitional dynamics substantially. Indeed, the strategic interaction not only leads to complex wealth distribution but also complex dynamics in Ramsey model with adjustment cost of consumption. The importance of these results is further emphasized if one recalls that the peculiar possibility of cyclical policies requires both extensions of the Ramsey model, a positive spillover of capital in utility and the adjustment costs of consumption (see Wirl, 1994; Wirl et al., 2008). However, we show that when households use open-loop strategies rather than being price takers, complex dynamics may emerge even without capital in utility at very low levels of adjustment costs. In this respect, we show that structurally very simple frameworks may lead to limit cycles thanks to the strategic interaction among agents in the economy.

(1984), Figueres et al. (1999), Dockner and Nishimura (2005) on capital accumulation games; Espino (2005), Bethmann (2008) on Lucas-Uzawa model; Sorger (2008) on Ramsey conjecture and Camacho, et al. (2013) on dynamics.

${ }^{2}$ Sorger (2008) proposes a strategic Ramsey model in which agents differ in their subjective time discount rate and analyzes Ramsey conjecture on the degeneracy of the long-run distribution of wealth. However, we assume that agents differ only in their initial wealth and analyze whether the poor can catch up with rich in the long run.

${ }^{3}$ Capitalist spirit refers to the motivation behind the perpetual acquisition of wealth not only for the sake of maximizing long-run consumption but also for the utility from accumulating wealth itself and the status associated with it (see Weber, 1958; Bakshi and Chen, 1996; Corneo and Jeanne, 1997). 
The rest of the paper is organized as follows. Section II describes the model and provides the dynamic properties of competitive and open-loop Nash equilibrium. Section III and IV consider the relative wealth concern and the adjustment costs of consumption, respectively. Finally, Section V concludes.

\section{MODEL}

We consider a Ramsey (1928) economy with $N \in \mathbb{N}$ infinitely lived households and a representative firm. The firm hires capital $K(t)$ and labor $L(t)$ from the households and produces a single output $Y(t)$ that can be either consumed or saved to form future capital. The technology is represented by a neoclassical production function $F: \mathbb{R}_{+}^{2} \rightarrow \mathbb{R}_{+}$. At any instant $t$, the firm chooses the variables $Y(t), K(t)$, and $L(t)$ to maximize the profit $Y(t)-w(t) L(t)-r(t) K(t)$ subject to the technology $Y(t)=F(K(t), L(t))$ and the nonnegativity constraints $K(t) \geq 0, L(t) \geq 0$ where $w(t)$ is the real wage rate and $r(t)$ is the rental rate of capital.

The preferences of household $i \in\{1,2, \ldots, N\}$ are characterized by the instantaneous utility function $u: \mathbb{R}_{+} \rightarrow \mathbb{R}$ and the time preference rate $\rho>0$. At any instant $t$, every household supply inelastically one unit of labor so that the total labor supply is $N$. Let $f(K(t))=F(K(t), N)$. We assume that $f$ and $u$ satisfy the following properties.

Assumption 1. $f: \mathbb{R}_{+} \rightarrow \mathbb{R}_{+}$, is continuous, twice continuously differentiable, strictly increasing, strictly concave satisfying $f(0)=0, \lim _{K \rightarrow 0} f^{\prime}(K)=+\infty$ and $\lim _{K \rightarrow+\infty} f^{\prime}(K)=$ 0 .

Assumption 2. $u: \mathbb{R}_{+} \rightarrow \mathbb{R}$, is continuous, twice continuously differentiable, strictly increasing, strictly concave satisfying $\lim _{c \rightarrow 0} u^{\prime}(c)=+\infty$.

The households differ only in terms of their initial wealth levels. Agents maximize their discounted lifetime utility derived from the consumption of the single good. The utility maximization problem of household $i$ can be formalized as

$$
\max _{c_{i}(t)} \int_{0}^{\infty} e^{-\rho t} u\left(c_{i}(t)\right) d t
$$

subject to

$$
\begin{gathered}
k_{i}(t)=r(t) k_{i}(t)+w(t)-c_{i}(t), \forall t \geq 0, \\
k_{i}(t) \geq 0, c_{i}(t) \geq 0, \forall t \geq 0, \\
k_{i}(0)=k_{i 0}, \text { given. }
\end{gathered}
$$

In what follows, we will analyze the competitive equilibrium and the open-loop Nash equilibrium in order to identify to what extent the strategic interaction among agents in the economy affects the long run distribution of wealth in terms of catching-up.

\section{II.1 Competitive equilibrium}

If we assume that the households are price-takers so that they can not realize their market power and take the rental rates of capital and labor as given, the model coincides with the standard Ramsey economy. Kemp and Shimomura (1992) and Van Long and Shimomura (2004) have already shown that the initial wealth inequality will persist in the long run so that the poor individuals will never be able to catch up with the rich in such a framework. For completeness 
and providing a basis of comparison, the analysis of competitive equilibrium follows from their studies.

The solution to the utility maximization problem $(\mathcal{P})$ of household $i \in\{1,2, \ldots, N\}$ leads to the following Euler equation:

$$
\frac{\dot{c}_{i}(t)}{c_{i}(t)}=\beta\left(c_{i}(t)\right)(r(t)-\rho),
$$

where $\beta\left(c_{i}\right) \equiv-\frac{u^{\prime}\left(c_{i}\right)}{c_{i} u^{\prime \prime}\left(c_{i}\right)}$ denotes the inverse of the elasticity of marginal utility.

Since the firm maximizes its profit taking the market prices as given, factors are paid their marginal products, i.e.,

$$
r(t)=f^{\prime}(K(t)) \text { and } w(t)=\frac{\left[f(K(t))-K(t) f^{\prime}(K(t))\right]}{N}, \forall t \geq 0,
$$

where $K(t)=\sum_{i=1}^{N} k_{i}(t)$. We have then the following system of $2 N$ differential equations:

$$
\begin{gathered}
\dot{k}_{i}(t)=f^{\prime}(K(t)) k_{i}(t)+\frac{f(K(t))-K(t) f^{\prime}(K(t))}{N}-c_{i}(t), \\
\frac{\dot{c}_{i}(t)}{c_{i}(t)}=\beta\left(c_{i}(t)\right)\left(f^{\prime}(K(t))-\rho\right), \forall i \in\{1,2, \ldots, N\} .
\end{gathered}
$$

A steady state is defined by $\left(k_{i}, c_{i}\right)$ such that the right-hand sides of the system of equations (2)-(3) equal to zero for all $i \in\{1,2, \ldots, N\}$. A steady state is said to be symmetric if $k_{i}=k$, and $c_{i}=c$, for all $i \in\{1,2, \ldots, N\}$. A steady state turns out to be asymmetric if $k_{i} \neq k_{j}$, for some $i, j \in\{1,2, \ldots, N\}$. The following proposition shows that if $k_{i}(0) \neq k_{j}(0)$ for some $i, j \in\{1,2, \ldots, N\}$ then we will have $k_{i} \neq k_{j}$ at the steady state.

Proposition 1. We have $k_{i}=k$, for all $i \in\{1,2, \ldots, N\}$ at the steady state if and only if the initial wealth levels are identical, i.e., $k_{i}(0)=k_{0}$, for all $i \in\{1,2, \ldots, N\}$. Moreover, there exists a continuum of steady state wealth distributions and a corresponding continuum of one-dimensional stable manifolds so that inequalities persist.

Proof. Follows directly from Kemp and Shimomura (1992) and Van Long and Shimomura (2004).

\section{II.2 Strategic Ramsey model and open loop Nash equilibrium}

Agents no longer act as price-takers but they take into account the effects of their accumulation decisions on market prices. Taking into account the inverse factor demand functions stated in (1), the households play a Nash equilibrium by choosing their capital paths. Households give their accumulation decisions as simple time paths and commit themselves to stick to these time paths during the entire game (i.e., they employ open-loop strategies). When choosing its path of capital, household $i \in\{1,2, \ldots, N\}$ takes the other households' choice variables as given. Accordingly, household $i$ solves the problem:

$$
\max _{c_{i}(t)} \int_{0}^{\infty} e^{-\rho t} u\left(c_{i}(t)\right) d t
$$


subject to

$$
\begin{gathered}
k_{i}(t)=f^{\prime}(K(t)) k_{i}(t)+\frac{f(K(t))-K(t) f^{\prime}(K(t))}{N}-c_{i}(t), \forall t \geq 0, \\
k_{i}(t) \geq 0, c_{i}(t) \geq 0, \forall t \geq 0, \\
k_{i}(0)=k_{i 0}, \text { given, } \\
k_{j}(t), \forall t \geq 0, \forall j \in\{1,2, \ldots, N\} \backslash\{i\}, \text { given. }
\end{gathered}
$$

It is important to note that household $i$ takes into account that it can influence $K(t)$ via $k_{i}(t)$ as $K(t)=\sum_{j=1}^{N} k_{j}(t)$. The Hamiltonian for problem $\mathcal{P}$ ' is:

$$
\begin{aligned}
H\left(c_{i}(t), k_{i}(t), \lambda_{i}(t)\right)= & e^{-\rho t} u\left(c_{i}(t)\right)+ \\
& \lambda_{i}(t)\left(f^{\prime}(K(t)) k_{i}(t)+\frac{f(K(t))-K(t) f^{\prime}(K(t))}{N}-c_{i}(t)\right) .
\end{aligned}
$$

The set of necessary conditions of optimality will then be written as follows: $\forall i \in$ $\{1,2, \ldots, N\}, \forall t \geq 0$,

$$
\begin{gathered}
e^{-\rho t} u^{\prime}\left(c_{i}(t)\right)=\lambda_{i}(t), \\
-\frac{\dot{\lambda_{i}}(t)}{\lambda_{i}(t)}=f^{\prime}(K(t))+f^{\prime \prime}(K(t))\left(k_{i}(t)-\frac{K(t)}{N}\right), \\
k_{i}(t)=f^{\prime}(K(t)) k_{i}(t)+\frac{f(K(t))-K(t) f^{\prime}(K(t))}{N}-c_{i}(t) .
\end{gathered}
$$

In order to make the first order optimality conditions for $\mathcal{P} /$ to be sufficient, we need to assume further that the factor income of each household is a concave function of its own capital stock. Assumption 3. The function $k_{i}(t) \mapsto k_{i}(t) f^{\prime}(K(t))$ is concave for all $i \in\{1, \ldots, N\}$ and for all $t \geq 0$.

Under Assumptions 1-3, the first order optimality conditions are also sufficient if the transversality condition $\lim _{t \rightarrow 0} \lambda_{i}(t) k_{i}(t)=0$ holds for all $i \in\{1, \ldots, N\}$. In accordance with Assumption 2, we adopt a $C R R A$ form of utility function with an intertemporal elasticity of substitution $\theta$ under which we obtain the following system of $2 N$ differential equations: $\forall i \in\{1,2, \ldots, N\}, \forall t \geq 0$,

$$
\begin{aligned}
& \frac{\dot{c}_{i}(t)}{c_{i}(t)}=\frac{1}{\theta}\left[f^{\prime}(K(t))-\rho+f^{\prime \prime}(K(t))\left(k_{i}(t)-\frac{K(t)}{N}\right)\right], \\
& k_{i}(t)=f^{\prime}(K(t)) k_{i}(t)+\frac{f(K(t))-K(t) f^{\prime}(K(t))}{N}-c_{i}(t) .
\end{aligned}
$$

In the following proposition, taking into account the strategic interaction among agents, we show that the catching up prevails in the economy, so that even if the agents have initially different levels of wealth, they will eventually reach equal levels of wealth at the steady state.

Proposition 2. In an open-loop Nash equilibrium, there exists a unique symmetric steady state and there are no asymmetric steady states.

Proof. Note from (B) that we have

$$
f^{\prime}(K)-\rho+f^{\prime \prime}(K)\left(k_{i}-\frac{K}{N}\right)=0, \forall i \in\{1,2, \ldots, N\}
$$


at a steady state. Since the production function is strictly concave, i.e., $f^{\prime}(K)>0, f^{\prime \prime}(K)<0$, the condition that satisfies these $N$ equations simultaneously is simply $k_{i}=k$, for all $i \in$ $\{1,2, \ldots, N\}$.

\section{II.3 Steady state and the stability analysis}

We now examine the stability properties of the symmetric steady state at which we have $f^{\prime}(K)=\rho, c_{i}=\frac{f(K)}{N}, \quad \forall i \in\{1,2, \ldots, N\}$, where $K=N k_{i}$. Linearizing the system of $2 N$ differential equations (7)-(8) around the unique steady state gives the following $2 N \times 2 N$ Jacobian matrix:

$$
\mathbf{J}_{2 N \times 2 N} \equiv\left[\begin{array}{ccc}
\mathbf{0}_{N \times N} & \vdots & \mathbf{A}_{N \times N} \\
\ldots \ldots & \cdots \cdots \\
-\mathbf{I}_{N \times N} & \vdots & f^{\prime}(K) \mathbf{I}_{N \times N}
\end{array}\right],
$$

where $\mathbf{I}$ and $\mathbf{0}$ denote the identity and zero matrix respectively and

$$
\mathbf{A}_{N \times N}=\frac{1}{\theta N} f(K) f^{\prime \prime}(K)\left[\begin{array}{cccc}
\left(2-\frac{1}{N}\right) & \left(1-\frac{1}{N}\right) & \cdots & \left(1-\frac{1}{N}\right) \\
\left(1-\frac{1}{N}\right) & \left(2-\frac{1}{N}\right) & \cdots & \left(1-\frac{1}{N}\right) \\
\vdots & \vdots & \ddots & \vdots \\
\left(1-\frac{1}{N}\right) & \left(1-\frac{1}{N}\right) & \cdots & \left(2-\frac{1}{N}\right)
\end{array}\right] .
$$

Note that $\mathbf{A}_{N \times N}$ is a symmetric Toeplitz (diagonal-constant) matrix with $(N-1)$ characteristic roots that are equal to $\frac{1}{\theta N} f(K) f^{\prime \prime}(K)$ and one characteristic root that is equal to $\frac{1}{\theta} f(K) f^{\prime \prime}(K)$. Without loss of generality, let $\mu_{1}=\mu_{2}=\ldots=\mu_{N-1}=\frac{1}{\theta N} f(K) f^{\prime \prime}(K)$ and $\mu_{N}=\frac{1}{\theta} f(K) f^{\prime \prime}(K)$. As $f^{\prime \prime}(K)<0$, we have $\mu_{i}<0, \forall i \in\{1,2, \ldots, N\}$.

To find the eigenvalues of the Jacobian matrix $\mathbf{J}$, we need to solve $\operatorname{det}[\mathbf{J}-\lambda \mathbf{I}]=0$. However, note that $\lambda$ is an eigenvalue of the jacobian matrix $\mathbf{J}$ if and only if $\lambda\left(f^{\prime}(K)-\lambda\right)$ is an eigenvalue of $\mathbf{A}_{N \times N}$ as

$$
\operatorname{det}[\mathbf{J}-\lambda \mathbf{I}]=\operatorname{det}\left[\mathbf{A}-\lambda\left(f^{\prime}(K)-\lambda\right) \mathbf{I}\right] .
$$

This simply suggests that the eigenvalues of the $2 N \times 2 N$ Jacobian matrix can easily be characterized by the characteristic root distribution of $\mathbf{A}_{N \times N}$. Indeed, the eigenvalues of the Jacobian matrix $\mathbf{J}$ will be determined as a solution to the quadratic equations,

$$
\lambda^{2}-f^{\prime}(K) \lambda+\mu_{i}=0, \forall i \in\{1,2, \ldots, N\} .
$$

In particular, for each eigenvalue $\mu_{i}$ of matrix $\mathbf{A}$, this equation has two roots, the product of which is $\mu_{i}<0$. Evidently, this implies that the Jacobian matrix $\mathbf{J}$ has $N$ positive and $N$ negative real eigenvalues so that the symmetric steady state turns out to be stable in the saddle-point sense.

\section{RELATIVE WEALTH EFFECT IN STRATEGIC RAMSEY MODEL}

Accepting that wealth is more valuable than its implied consumption rewards, Van Long and Shimomura (2004) consider that the agents get utility not only from their consumption stream but also from their relative wealth level with respect to the average in the economy. Thanks to this relative wealth effect in utility, Van Long and Shimomura (2004) show that the poor will be able to catch up with the rich if the elasticity of the marginal utility of relative wealth is greater 
than the elasticity of the marginal utility of consumption. However, even though the agents take care of their relative wealth position in the economy so that the strategic interaction among agents inherently exists, this has not been taken into account in assessing the conclusions on catching up.

The model differs from the strategic Ramsey model by the assumption on the preference of the agents. In this set up, households take utility not only from their consumption but also from their social status represented by their relative wealth. The maximization problem of household $i \in\{1,2, \ldots, N\}$ can now be formalized as follows:

$$
\underset{c_{i}(t)}{\operatorname{Max}} \int_{0}^{\infty} e^{-\rho t}\left(u\left(c_{i}(t)\right)+\eta_{i} v\left(z_{i}(t)\right)\right) d t
$$

subject to

$$
\begin{gathered}
\dot{k}_{i}(t)=f^{\prime}(K(t)) k_{i}(t)+\frac{f(K(t))-K(t) f^{\prime}(K(t))}{N}-c_{i}(t), \forall t \geq 0, \\
z_{i}(t)=\frac{k_{i}(t)}{\frac{K(t)}{N}, \forall t \geq 0,} \\
k_{i}(t) \geq 0, c_{i}(t) \geq 0, \forall t \geq 0, \\
k_{i}(0)=k_{i 0}, \text { given, } \\
k_{j}(t), \forall t \geq 0, \forall j \in\{1,2, \ldots, N\} \backslash\{i\}, \text { given, }
\end{gathered}
$$

where $z_{i}$ refers to the relative wealth of household $i$ with respect to the average wealth in the economy. $\eta_{i} \in \mathbb{R}_{+}$measures the weight of relative wealth (status concern) in utility. We employ an additively separable utility function between consumption and realtive wealth not only for analytical convenience but also for being consistent with Van Long and Shimomura (2004) and the recent empirical findings. ${ }^{4}$ We adopt the following assumption on the utility from relative wealth.

Assumption 4. $\quad v: \mathbb{R}_{+} \rightarrow \mathbb{R}$, is continuous, twice continuously differentiable, strictly increasing, strictly concave satisfying $v(0)=0$ and $\lim _{z \rightarrow 0} v^{\prime}(z)=+\infty$.

The current-value Hamiltonian associated with optimization problem $\mathcal{P}^{\prime \prime}$ is

$$
\begin{aligned}
\mathcal{H}\left(c_{i}(t), k_{i}(t), \lambda_{i}(t)\right)= & {\left[u\left(c_{i}(t)\right)+\eta_{i} v\left(\frac{k_{i}(t)}{\frac{1}{N} K(t)}\right)\right]+} \\
& \lambda_{i}(t)\left[f^{\prime}(K(t)) k_{i}(t)+\frac{f(K(t))-K(t) f^{\prime}(K(t))}{N}-c_{i}(t)\right],
\end{aligned}
$$

where $\lambda_{i}$ denotes the current-value adjoint variable. Recall that household $i$ takes into account that it can influence $K(t)$ via $k_{i}(t)$ as $K(t)=\sum_{j=1}^{N} k_{j}(t)$. A routine application of the Pontryagin's maximum principle leads to the following system of $2 N$ differential equations: $\forall i \in\{1,2, \ldots, N\}, \forall t \geq 0$,

$$
\dot{c}_{i}(t)=-\frac{u^{\prime}\left(c_{i}(t)\right)}{u^{\prime \prime}\left(c_{i}(t)\right)}\left[f^{\prime}(K(t))-\rho+f^{\prime \prime}(K(t))\left(k_{i}(t)-\frac{K(t)}{N}\right)+\eta_{i} \frac{v^{\prime}\left(z_{i}(t)\right)}{u^{\prime}\left(c_{i}(t)\right)} \frac{1-\frac{k_{i}(t)}{K(t)}}{\frac{1}{N} K(t)}\right],
$$

\footnotetext{
${ }^{4}$ Compared to the multiplicative form, the separable form of the preferences is more consistent with the empirical findings on the behavior of the wealthy households since these preferences do not put any restrictions on either the substitutability or the complementarity between consumption and relative wealth (see Francis (2009) for details about the functional form of the utility function).
} 


$$
\dot{k}_{i}(t)=f^{\prime}(K(t)) k_{i}(t)+\frac{f(K(t))-K(t) f^{\prime}(K(t))}{N}-c_{i}(t) .
$$

Under Assumptions 1-4, the first order optimality conditions are also sufficient if the transversality condition $\lim _{t \rightarrow 0} e^{-\rho t} \lambda_{i}(t) k_{i}(t)=0$ holds for all $i \in\{1, \ldots, N\}$.

Taking into account the strategic interaction among agents, we now consider the conditions under which catching up will prevail in the economy. It is clear from equations (9)-(10) that $\eta_{i}=\eta$ for all $i \in\{1, \ldots, N\}$ turns out to be a necessary condition for the existence of a symmetric steady state. Indeed, given $\eta_{i}=\eta$, for all $i \in\{1, \ldots, N\}$, a symmetric steady state exists if and only if there exists $K>0$ such that

$$
f^{\prime}(K)+\eta^{1} \frac{v^{\prime}(1)}{u^{\prime}\left(\frac{f(K)}{N}\right)} \frac{(N-1)}{K}=\rho .
$$

In accordance with Assumption 2 and 4, adopting $C R R A$ form of utility functions, $u(c)=$ $\frac{c^{1-\theta}}{1-\theta}$ and $v(z)=\frac{z^{1-\sigma}}{1-\sigma}$, and the standard Cobb-Douglas production function $f(K)=A K^{\gamma}, \gamma \in$ $(0,1)$, the following proposition provides the conditions for the existence and uniqueness of a symmetric steady state.

Proposition 3. Let $\eta_{i}=\eta$, for all $i \in\{1, \ldots, N\}$. There exists a unique symmetric steady state if $\gamma \theta<1$. Moreover, if $\theta \leq \sigma$ then an asymmetric steady state does not exist.

Proof. See Van Long and Shimomura (2004) for the existence and the uniqueness of a symmetric steady state. The proof of the existence of a symmetric steady state follows from the limit properties of the left-hand side of equation (11). Given existence, if $\left[K u^{\prime}(f(K))\right]$ is monotonically decreasing in $K$ then the uniqueness of the symmetric steady state is also ensured.

Assume now that there exists an asymmetric steady state at which $k_{i}>k_{j}$, for some $i, j \in$ $\{1, \ldots, N\}$, without loss of generality. Let $c_{i}$ and $c_{j}$ denote the associated consumption levels at this asymmetric steady state. It is then clear from (9) and (10) that

$$
f^{\prime \prime}(K)\left(k_{i}-k_{j}\right)=\frac{N \eta}{K^{2}}\left(\frac{v^{\prime}\left(z_{j}\right)}{u^{\prime}\left(c_{j}\right)}\left(K-k_{j}\right)-\frac{v^{\prime}\left(z_{i}\right)}{u^{\prime}\left(c_{i}\right)}\left(K-k_{i}\right)\right) .
$$

Note that the left-hand side of (12) is less than zero due to the concavity of the production function. However, since $\theta \leq \sigma$, we have

$$
\left(\frac{z_{j}}{z_{i}}\right)^{-\sigma} \geq\left(\frac{c_{j}}{c_{i}}\right)^{-\theta}
$$

as

$$
\left(\frac{k_{i}}{k_{j}}\right)^{\sigma} \geq\left(\frac{k_{i}}{k_{j}}\right)^{\theta} \geq\left(\frac{f^{\prime}(K) k_{i}+\frac{f(K)-K f^{\prime}(K)}{N}}{f^{\prime}(K) k_{j}+\frac{f(K)-K f^{\prime}(K)}{N}}\right)^{\theta},
$$

so that the right-hand side of (12) is positive: a contradiction.

If initially poor households attribute less weight to the relative wealth (status concern) in utility than the initially rich households, then the poor can never catch up with the rich in such a strategic Ramsey economy. Note that, as we focus on catching up, we need also the condition that the elasticity of consumption is less than that of relative wealth for avoiding the emergence of the asymmetric steady states. Moreover, we also need to verify that the unique symmetric steady state turns out to be stable at least in the saddle-point sense. 
To do so, we characterize the Jacobian of the resulting $2 N \times 2 N$ system associated with (III) around a symmetric steady state $\left(c_{i}, k_{i}\right)_{i \in\{1, \ldots, N\}}$ at which $\eta_{i}=\eta, c_{i}=c=\frac{f(K)}{N}, k_{i}=k$ for all $i \in\{1, \ldots, N\}$, and $K=N k$ satisfy (11). The Jacobian is a $2 \times 2$ block (partitioned) matrix,

$$
\mathbf{J}_{2 N \times 2 N} \equiv\left[\begin{array}{ccc}
\left(\rho-f^{\prime}(K)\right) \mathbf{I}_{N \times N} & \vdots & \mathbf{B}_{N \times N} \\
\ldots \ldots & \ldots \ldots \\
-\mathbf{I}_{N \times N} & \vdots & f^{\prime}(K) \mathbf{I}_{N \times N}
\end{array}\right],
$$

at which

$$
\mathbf{B}_{N \times N}=\left[\begin{array}{cccc}
a & b & \cdots & b \\
b & a & \ddots & b \\
\vdots & \ddots & \ddots & b \\
b & \cdots & b & a
\end{array}\right],
$$

is a symmetric Toeplitz (diagonal-constant) matrix with $(N-1)$ characteristic roots that are equal to $(a-b)$ and one characteristic root that is equal to $((N-1) b+a)$ where

$$
\begin{aligned}
& a=\frac{c}{\theta}\left(f^{\prime \prime}(K)\left(2-\frac{1}{N}\right)+\eta \frac{v^{\prime}(1)}{u^{\prime}(c)}\left(\frac{N-1}{K}\right)^{2}\left(\frac{v^{\prime \prime}(1)}{v^{\prime}(1)}-\frac{2}{N-1}\right)\right), \\
& b=\frac{c}{\theta}\left(f^{\prime \prime}(K)\left(1-\frac{1}{N}\right)-\eta \frac{v^{\prime}(1)}{u^{\prime}(c)} \frac{N-1}{K^{2}}\left(\frac{v^{\prime \prime}(1)}{v^{\prime}(1)}-\frac{2-N}{N-1}\right)\right) .
\end{aligned}
$$

Since

$$
\operatorname{det}[\mathbf{J}-\lambda \mathbf{I}]=\operatorname{det}\left[\mathbf{B}-\left(\lambda-f^{\prime}(K)\right)\left(\rho-f^{\prime}(K)-\lambda\right) \mathbf{I}\right],
$$

the eigenvalues of the $2 N \times 2 N$ Jacobian matrix can easily be characterized by the characteristic roots $\mu_{i}, i \in\{1,2, \ldots, N\}$ of $\mathbf{B}_{N \times N}$. Indeed, the eigenvalues of the Jacobian matrix $\mathbf{J}$ will be determined as a solution to the quadratic equations,

$$
\lambda^{2}-\rho \lambda+\left(f^{\prime}(K)\left(\rho-f^{\prime}(K)\right)+\mu_{i}\right)=0, \forall i \in\{1,2, \ldots, N\},
$$

where

$$
\mu_{i}=\left\{\begin{array}{l}
\frac{c}{\theta}\left(f^{\prime \prime}(K)+\eta \frac{v^{\prime}(1)}{u^{\prime}(c)} \frac{N(N-1)}{K^{2}}\left(\frac{v^{\prime \prime}(1)}{v^{\prime}(1)}-\frac{1}{N-1}\right)\right), \forall i \in\{1,2, \ldots, N-1\}, \\
\frac{c}{\theta}\left(f^{\prime \prime}(K) N-\eta \frac{v^{\prime}(1)}{u^{\prime}(c)} \frac{N(N-1)}{K^{2}}\right), i=N .
\end{array}\right.
$$

In particular, for each eigenvalue $\mu_{i}$ of matrix $\mathbf{B}$, this equation has two roots, the product of which equals to $\left(f^{\prime}(K)\left(\rho-f^{\prime}(K)\right)+\mu_{i}\right)$. Recalling that $\gamma \theta<1, \theta \leq \sigma$, and we have (11) at a unique symmetric steady state, one can easily show that the product of the two roots is less than zero. This implies that the Jacobian matrix $\mathbf{J}$ has $N$ positive and $N$ negative real eigenvalues which reveals saddle path stability with monotone convergence.

It is already clear that, the long run distribution of wealth heavily depends on the valuation of the relative position by the initially poor and the rich households. Indeed, as soon as $\eta_{i}$ differs from $\eta_{j}$ for some $i, j \in\{1,2, \ldots, N\}$, the strategic Ramsey model with relative wealth concern would result with a complex wealth distribution characterized by a saddle path stable asymmetric steady state. The next section is devoted to the analysis of the strategic Ramsey model with adjustment costs of consumption under which not only complex wealth distribution but also complex dynamics may emerge. 


\section{ADJUSTMENT COST OF CONSUMPTION IN STRATEGIC RAMSEY MODEL}

The strategic Ramsey model is now extended for considering the dynamic implications of consumption adjustment costs by assuming that the agents not only derive utility from consumption but also incur a disutility from the adjustments of consumption. Accordingly, the problem of household $i \in\{1,2, \ldots, N\}$ recast as follows:

$$
\underset{c_{i}(t)}{\operatorname{Max}} \int_{0}^{\infty} e^{-\rho t}\left\{u\left(c_{i}(t)\right)-\Omega\left(\kappa_{i}(t)\right)\right\} d t
$$

subject to

$$
\begin{gathered}
\dot{k}_{i}(t)=f^{\prime}(K(t)) k_{i}(t)+\frac{f(K(t))-K(t) f^{\prime}(K(t))}{N}-c_{i}(t), \forall t \geq 0, \\
\dot{c}_{i}(t)=\kappa_{i}(t), \forall t \geq 0, \\
k_{i}(t) \geq 0, c_{i}(t) \geq 0, \forall t \geq 0, \\
k_{i}(0)=k_{i 0}, \text { given, } \\
k_{j}(t), \forall t \geq 0, \forall j \in\{1,2, \ldots, N\} \backslash\{i\}, \text { given. }
\end{gathered}
$$

The additional state equation and the account for the adjustment costs $\left(\Omega\left(\kappa_{i}(t)\right)\right)$ constitute an important extension to the strategic Ramsey model. We adopt the following assumption on the adjustment cost function.

Assumption 5. $\Omega: \mathbb{R} \rightarrow \mathbb{R}$, is continuous, twice continuously differentiable, strictly increasing, strictly convex and $\Omega^{\prime}($.$) is invertible.$

The current-value Hamiltonian associated with the optimization problem $\mathcal{P}^{\prime \prime \prime}$ writes as

$$
\begin{aligned}
& H\left(c_{i}(t), k_{i}(t), \kappa_{i}(t), \lambda_{i}(t), \mu_{i}(t)\right)=U\left(c_{i}(t)\right)-\Omega\left(\kappa_{i}(t)\right)+ \\
& \quad \lambda_{i}(t)\left(f^{\prime}(K(t)) k_{i}(t)+\frac{f(K(t))-K(t) f^{\prime}(K(t))}{N}-c_{i}(t)\right)+\mu_{i}(t) \kappa_{i}(t) .
\end{aligned}
$$

The application of the Pontryagin's maximum principle leads to the following system of $4 \mathrm{~N}$ differential equations: $\forall i \in\{1,2, \ldots, N\}, \forall t \geq 0$,

$$
\begin{gathered}
\dot{\mu}_{i}(t)=\mu_{i}(t) \rho+\lambda_{i}(t)-U^{\prime}\left(c_{i}(t)\right), \\
\dot{\lambda}_{i}(t)=\lambda_{i}(t) \rho-\lambda_{i}(t)\left(f^{\prime}\left(K(t)+\Theta\left(k_{i}(t)\right)\right),\right. \\
\dot{c}_{i}(t)=\Lambda\left(\mu_{i}(t)\right), \\
\dot{k}_{i}(t)=f^{\prime}(K(t)) k_{i}(t)+\frac{f(K(t))-K(t) f^{\prime}(K(t))}{N}-c_{i}(t),
\end{gathered}
$$

where $\Theta\left(k_{i}(t)\right)=f^{\prime \prime}(K(t))\left(k_{i}(t)-\frac{K(t)}{N}\right)$. Note from $(15)$ that $\Lambda^{\prime}\left(\mu_{i}(t)\right)=\frac{1}{\Omega^{\prime \prime}\left(k_{i}(t)\right)}$ by the implicit function theorem. ${ }^{5}$ Under Assumptions 1-5, the first order optimality conditions are not only necessary but also sufficient if the transversality conditions, $\lim _{t \rightarrow 0} e^{-\rho t} \lambda_{i}(t) k_{i}(t)=0$, and $\lim _{t \rightarrow 0} e^{-\rho t} \mu_{i}(t) c_{i}(t)=0$ hold for all $i \in\{1, \ldots, N\}$.

${ }^{5} \mu_{i}(t)=\Omega^{\prime}\left(\kappa_{i}(t)\right)$ and as $\Omega^{\prime}($.$) is invertable, then \Lambda\left(\mu_{i}(t)\right)=\kappa_{i}(t)$. Thus, $\Omega^{\prime}\left(\Lambda\left(\mu_{i}(t)\right)\right)-\mu_{i}(t)=0$. The first derivative will give the result. 
A steady state is defined by $\left(\mu_{i}, \lambda_{i}, k_{i}, c_{i}\right)$ such that the right-hand sides of the system of differential equations (13)-(16) equal to zero for all $i \in\{1,2, \ldots, N\}$. The following proposition is devoted to the existence and uniqueness of a symmetric steady state.

Proposition 4. There exists a unique symmetric steady state and there are no asymmetric steady states in the strategic Ramsey model augmented with the adjustment cost of consumption.

Proof. Note from (14) that $\dot{\lambda}_{i}(t)=0$ for all $i \in\{1, \ldots, N\}$ if and only if $\Theta\left(k_{i}\right)=$ $\rho-f^{\prime}(K)$, for all $i \in\{1,2, \ldots, N\}$. We have then $f^{\prime \prime}(K)\left(k_{i}-\frac{K}{N}\right)=f^{\prime \prime}(K)\left(k_{j}-\frac{K}{N}\right)$ for all $i, j \in\{1,2, \ldots, N\}, i \neq j$. As $f^{\prime \prime}<0$, this implies $k_{i}=\frac{K}{N}$, for all $i \in\{1,2, \ldots, N\}$ so that the emergence of an asymmetric steady state is ruled out. The existence of a unique symmetric steady state then follows easily from the monotonicity and the limit properties of the right-hand side of equation (14).

It is important to note that the model with adjustment cost of consumption reduces to the standard Ramsey model at the steady state thanks to the price taking assumption of the competitive equilibrium. Because of this, the qualitative properties of the competitive equilibrium of the standard Ramsey model will be carried over to the Ramsey model augmented with the Easterlin hypothesis. Accordingly, as Van Long and Shimomura (2004) have shown for the standard Ramsey model, there exists a continuum of steady state wealth distributions and a corresponding continuum of one-dimensional stable manifolds in the Ramsey model augmented with consumption adjustment costs. In other words, the Ramsey model augmented with consumption adjustment costs predicts that the initial wealth differences will continue to persist in a competitive equilibrium environment.

However, to what extent the qualitative features of the strategic Ramsey model will carry over to the strategic Ramsey model with adjustment cost of consumption is not yet clear. Even though, we show that there exists a symmetric steady state, we need to analyze the dynamic properties of the associated system as well. The next section presents that the strategic interaction may induce cycles à la Hopf in the strategic Ramsey model with consumption adjustment costs.

\section{IV.1 Hopf bifurcation and Easterlin cycles}

The analysis follows from Guckenheimer et al. (1997) that serves procedures for locating Hopf bifurcations in any $n$-dimensional system of ordinary differential equations based on the singularity of matrices stemming from algebraic transformations of the Jacobian at a steady state.

In what follows, for the sake of dimensional simplicity, we consider an economy with $N=2$ households which differ in terms of initial level of capital stock. Recall from the system of differential equations (13)-(16) that we have now a system of eight differential equations in terms of capital and consumption levels of the poor and the rich household. Let $J$ denote the Jacobian of this system of equations around the unique symmetric steady state $\left(c^{*}, k^{*}, \lambda^{*}, \mu^{*}\right)$ and let $p(\omega)$ be the associated characteristic polynomial so that

$$
p(\omega)=a_{0}+a_{1} \omega+a_{2} \omega^{2}+\cdots \cdots+a_{7} \omega^{7}+a_{8} \omega^{8} .
$$

$p(\omega)$ has the non-zero root pair $\{\omega,-\omega\}$ if and only if $\omega$ is a common root of the two equations $p(\omega)+p(-\omega)=0$ and $p(\omega)-p(-\omega)=0$. Substituting $z=\omega^{2}$, construct two new polynomials:

$$
r_{e}(z)=a_{0}+a_{2} z+a_{4} z^{2}+a_{6} z^{3}+z^{4}
$$




$$
r_{o}(z)=a_{1}+a_{3} z+a_{5} z^{2}+a_{7} z^{3} .
$$

Then $p$ has a non-zero root pair $\{\omega,-\omega\}$ if there exist a $z$ that satisfies $\left(\begin{array}{c}r_{e}(z) \\ r_{o}(z)\end{array}\right)=0$. Let the Sylvester matrix of the pair of equations (17) and (18) be the $7 \times 7$ - matrix given by

$$
S=\left[\begin{array}{ccccccc}
a_{0} & a_{2} & a_{4} & a_{6} & 1 & 0 & 0 \\
0 & a_{0} & a_{2} & a_{4} & a_{6} & 1 & 0 \\
0 & 0 & a_{0} & a_{2} & a_{4} & a_{6} & 1 \\
a_{1} & a_{3} & a_{5} & a_{7} & 0 & 0 & 0 \\
0 & a_{1} & a_{3} & a_{5} & a_{7} & 0 & 0 \\
0 & 0 & a_{1} & a_{3} & a_{5} & a_{7} & 0 \\
0 & 0 & 0 & a_{1} & a_{3} & a_{5} & a_{7}
\end{array}\right]
$$

For $m \in\{0,1\}$, let $S_{m}$ denote the matrix obtained from $S$ by deleting the rows 1 and 4 and the columns 1 and $m+2$. By means of the relation between the characteristic polynomial and its corresponding matrices $S, S_{0}, S_{1}$, Guckenheimer, et al. (1996) provides the following result.

Theorem 1. (Guckenheimer et al., 1997) Let $S$ be the Sylvester matrix for polynomials $r_{e}$ and $r_{o}$. Then $J$ has precisely one pair of pure imaginary eigenvalues if $\operatorname{det}[S]=0$ and $\operatorname{det}\left[S_{0}\right] \operatorname{det}\left[S_{1}\right]>0$. If $\operatorname{det}[S] \neq 0$ or $\operatorname{det}\left[S_{0}\right] \operatorname{det}\left[S_{1}\right]<0$, then $p(\omega)$ has no purely imaginary roots.

This procedure for locating Hopf bifurcations is specifically designed to locate points at which a simple pair of pure imaginary eigenvalues for the Jacobian of the system. When the conditions of the Theorem 1 are satisfied, the magnitudex of the shared root, $\sqrt{\operatorname{det}\left[S_{1}\right] / \operatorname{det}\left[S_{0}\right]}$ can be easily related to the period of the limit cycle created at the Hopf bifurcation point.

Then the main task is to show a parameter constellation under which the implementation of Theorem 1 yields a pair of purely imaginary eigenvalues. To do so, we adopt the standard $C o b b$ Douglas production function, $f(K)=A K^{\gamma}$ and $C R R A$ form of utility from consumption, $u(c)=\frac{c^{1-\theta}}{1-\theta}$. Following Wirl (1996), the adjustment cost of consumption is represented by a convex second degree penalty function, $\Omega\left(\kappa_{i}(t)\right)=\frac{1}{2} \psi \kappa_{i}(t)^{2}$. We consider the following fairly standard values for the parameters:

$$
A=1, \quad \rho=0.03, \quad \theta=0.8, \quad \gamma=0.3,
$$

under which the unique symmetric steady state of the economy reveals: $k^{*}=13.4135$. Since the parameter $\psi$ does not affect the level of the unique symmetric steady state, it turns out to be an ideal bifurcation parameter. Indeed, by Theorem 1 , setting $\psi=1.3 \times 10^{-3}$ yields precisely one pair of pure imaginary eigenvalues. This pair of purely imaginary roots is important as it facilitates the existence of limit cycles in the parameter domain of locally unstable spirals for those parameters that are close to the bifurcation point. ${ }^{6}$

Some remarks on the existence of limit cycles due to the strategic interaction are in order. First, it must be noted that the number of agents in the economy does not bring any qualitative change in the result. Second, it is important to note that complex dynamics emerge even with the separability of objective function in $\mathcal{P}^{\prime \prime \prime}$ contrary to the earlier attempts to explain cyclical patterns (see e.g., Dockner and Feichtinger, 1993). Third, recall that the competitive equilibrium of the Ramsey model augmented with consumption adjustment costs leads to a unique asymmetric steady state that is saddle-path sable under these parameter values. This

\footnotetext{
${ }^{6}$ Indeed, Hopf bifurcation theorem ensures the existence of limit cycles, if a pair of purely imaginary eigenvalues exist and if the velocity when crossing the imaginary axis is nonzero. See Guckenheimer and Holmes, 1983.
} 
reveals that the initially poor household can never catch up with the rich. However, deviating from the competitive equilibrium by taking into account the strategic interaction among agents in the economy not only leads to complex wealth distribution but also complex dynamics as well. Put differently, the strategic interaction brings out the emergence of Hopf bifurcation that leads to limit cycles in Ramsey model with consumption adjustment costs. The emergence of such cyclical patterns of consumption could provide an alternative source for explaining real business cycles (see e.g., Wirl et al., 2008).

The last but not the least, the importance of these results is further emphasized if one recalls that sufficiently large consumption adjustment costs may induce complex, in particular, cyclical policies if there exist positive contributions of capital to utility in an optimal growth framework (see e.g., Wirl, 1994). However, we show that when households use open-loop strategies rather than being price takers, complex dynamics may emerge even without capital in utility at very low levels of adjustment costs. In this respect, we show that structurally very simple frameworks may lead to limit cycles thanks to the strategic interaction among agents in the economy.

\section{CONCLUSION}

Considering the strategic interaction among agents changes the qualitative properties of the standard Ramsey model. In the absence of strategic interaction, the poor will never be able to catch up with the rich as pointed out in Van Long and Shimomura (2004). However, incorporating the strategic behavior among agents leads to the wealth level of the two classes to be the same at the stationary state. Extending the analysis to account for relative wealth concern and the adjustment cost of consumption, the strategic interaction among agents is shown to lead to a change not only in the distribution of wealth in the long run but also in the transitional dynamics substantially. In particular, the strategic interaction leads to the emergence of Hopf bifurcation that reveals limit cycles in the Ramsey model with consumption adjustment costs.

This paper can be extended to incorporate closed-loop strategies that allow agents to reconsider their strategy during the entire game and analyze the long-run distribution of wealth in terms of catching-up under Markov perfect equilibria.

\section{REFERENCES}

Baskshi, G. S. and Chen, Z. (1996). 'The spirit of capitalism and stock-market prices', The American Economic Review, 86(1), pp. 133-57.

Camacho, C., Saglam, C. and Turan, A. (2013). 'Strategic interaction and dynamics under endogenous time preference'. Journal of Mathematical Economics, 49(4), pp. 291-391.

Bethmann, D. (2008). 'The open-loop solution of the Uzawa-Lucas model of endogenous growth with N agents'. Journal of Macroeconomics, 30, pp. 396-414.

Corneo, G. and Jeanne, O. (2001). 'The distribution of wealth and growth', Scandinavian Journal of Economics, 103, pp. 283-93.

Dockner, E. J. and Feichtinger, G. (1993). 'On the optimality of limit cycles in dynamic economic systems', Journal of Economics, 53(1), pp. 31-50.

Dockner, E. J. and Nishimura, K. (2005). 'Capital accumulation games with a non-concave production function', Journal of Economic Behaviour and Organization, 57, pp. 408-20.

Easterlin, R. A. (2005). 'Income and happiness: towards a unified theory', The Economic Journal, 111(473), pp. 465-84.

Espino, E. (2005). 'On Ramsey conjecture: efficient allocations in the neoclassical growth model with private information', Journal of Economic Theory, 121, pp. 192-213. 
Fershtman, C. and Muller, E. (1984). 'Capital accumulation games of infinite duration', Journal of Economic Theory, 33, pp. 322-39.

Figuiered, C., Garderes, P., Michel, P. and Rychen, F. (1999). 'The dynamics of the strategic capital accumulation', Annals of Operations Research, 88, pp. 291-307.

Francis, J. L. (2009). 'Wealth and capitalist spirit', Journal of Macroeconomics, 31(3), pp. 394-408.

Guckenheimer, J. and Holmes, P. (1983). Nonlinear oscillations, dynamical systems and bifurcations of vector fields, New York: Springer.

Guckenheimer, J., Myers, M. and Sturmfels, B. (1997). 'Computing Hopf bifurcations I. SIAM', Journal of Numerical Analysis, 34(1), 1-21.

Hof, F. X., Novak, A. J. and Wirl, F. (2008). 'Happiness due to consumption and its increases, wealth and status', Studies in Nonlinear Dynamics and Econometrics, 12(4), 1567.

Kemp, M. C. and Shimomura, K. A. (1992). 'A dynamic model of the distribution of wealth among household and nations', Annals of Operation Research, 37, pp. 245-72.

Pichler, P. and Sorger, G. (2009). 'Wealth distribution and aggregate time-preference: Markovperfect equilibria in a Ramsay economy', Journal of Economic Dynamics and Control, 33, pp. $1-14$.

Sorger, G. (2002). 'On the long run distribution of capital in the Ramsey model', Journal of Economic Theory, 105(1), pp. 226-43.

Sorger, G. (2008). 'Strategic saving decisions in the infinite-horizon model', Economic Theory, 36 , pp. 353-77.

Ramsey, F. P. (1928). 'A mathematical theory of saving', Economic Journal, 37, pp. 47-61.

Stiglitz, J. E. (1969). 'Distribution of income and wealth among individuals', Econometrica, 37 (3), pp. 382-97.

Van Long, N. and Shimomura, K. (2004). 'Relative wealth, status seeking and catching up', Journal of Economic Behavior and Organization, 53, pp. 529-54.

Weber, M. (1958). The protestant ethic and the spirit, New York: Charles Scribner's Sons.

Wirl, F. (1994). 'The Ramsey model revisited: the optimality of cyclical consumption and growth', Journal of Economics, 60, 81-98.

Wirl, F. (1996). 'Pathways to Hopf birfurcations in dynamic continuous time optimization problems', Journal of Optimization Theory and Applications, 81, pp. 299-320. 